\title{
A Reaction Kinetic Model for Vacuum-Field Catalysis Based on Vibrational Light- Matter Coupling
}

\author{
Hidefumi Hiura*1 and Atef Shalabney ${ }^{2}$ \\ ${ }^{1}$ System Platform Research Laboratories, NEC Corporation, 34 Miyukigaoka, Tsukuba, Ibaraki 305-8501 \\ ${ }^{2}$ Physics and Optical Engineering Department, Braude College, Snunit St 51, Karmiel, 2161002, Israel.
}

\begin{abstract}
Since conventional catalysts are materials-based, they are effective only for particular chemical reactions. Recent studies suggest that vacuum-field catalysis (or cavity catalysis) based on vibrational light-matter coupling can boost reactions without the above constraint. Herein, we propose a reaction kinetic model for such vacuum-field-catalyzed reactions. Vibrational lightmatter coupling is an interaction in which a molecular vibration and infrared (IR) vacuum field are coupled in resonance, consequently creating a pair of Rabi-split vibro-polaritonic states. Our kinetic model hypothesizes that vibrational light-matter coupling reshapes the reaction potential surface, thereby changing its reaction barrier height. We translate such a qualitative picture into two kinds of analytical equations derived from the Arrhenius and Eyring-Polanyi theories: both the equations are obtained as a function of the coupling ratio $\Omega_{R} / 2 \omega_{0}$ of vibro-polaritons $\left(\Omega_{\mathrm{R}}\right.$ : Rabi frequency between a pair of vibropolaritons, $\omega_{0}$ : vibrational frequency of reactants), indicating that $\Omega_{\mathrm{R}} / 2 \omega_{0}$ is a decisive quantity to define the catalytic activity of vacuum-field catalysis. Our numerical calculation shows that when $\Omega_{\mathrm{R}} / 2 \omega_{0} \geq 0.1$, reactions may be accelerated by several orders of magnitude. Most importantly, our kinetic model can account well for rate enhancements ranging from $\sim 10^{0}$ to $\sim 10^{4}$ observed for vacuum-field-catalyzed reactions. We expect that our findings will bring fresh perspectives not only to chemistry but also to the broad fields of science and technology.
\end{abstract}

\section{Introduction}

Over the past quarter of a century, strong and ultra strong coupling between light and matter has been realized for a number of chemical and physical systems and used to create half-matter and half-light quantum states with novel properties. ${ }^{1,2}$ Such studies in light-matter interactions open up brand-new fields of science, as reviewed recently. ${ }^{1-4}$ In 2015, Shalabney et al. demonstrated vibrational strong coupling (V-SC) between the $\mathrm{C}=\mathrm{O}$ stretching mode in solid poly(vinyl acetate) and infrared (IR) vacuum fields (electromagnetic zero-point field of the quantum vacuum) $)^{3,4}$ in a Fabry-Pérot (FP) cavity, with a Rabi frequency $\Omega_{\mathrm{R}}$ of $167 \mathrm{~cm}^{-1} .{ }^{5}$ As shown in Fig. 1, V-SC is a lightmatter interaction in which the vibrational mode of molecules is resonantly coupled with the optical mode of an IR cavity in such a way that $\Omega_{R}$ can exceed the sum of the loss rates of the vibrational and optical modes. ${ }^{1-4}$ To date, V-SC has been observed for many molecular systems with FP cavities ${ }^{6-19}$ and investigated theoretically ${ }^{20-32}$ to understand the fundamental properties of V-SC. George et al. reported vibrational ultrastrong coupling (V-USC) for the first time for degenerate $\mathrm{C} \equiv \mathrm{O}$ stretching modes of liquid iron pentacarbonyl $\mathrm{Fe}(\mathrm{CO})_{5}\left(\Omega_{\mathrm{R}}=\right.$ $480 \mathrm{~cm}^{-1}$ ) with multiple Rabi splittings. ${ }^{9}$ More recently, giant Rabi splitting under V-USC was found in water and ice ${ }^{17,33}$ with $\Omega_{\mathrm{R}}=740 \mathrm{~cm}^{-1}$ for the $\mathrm{OH}$ stretching mode of $\mathrm{H}_{2} \mathrm{O}$ water and $\Omega_{\mathrm{R}}$ $=820 \mathrm{~cm}^{-1}$ for the $\mathrm{OH}$ stretching mode of $\mathrm{H}_{2} \mathrm{O}$ ice. These values of $\Omega_{R}$ are the largest ever reported in the literature. (a)

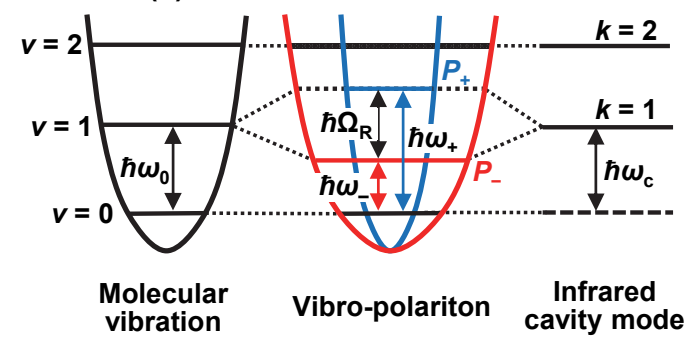

Figure 1. Energy diagrams of vibrational light-matter coupling: (a) molecular vibration, (b) vibro-polariton, (c) IR cavity mode.

The most recent studies of vibrational light-matter coupling have focused on polaritonic chemistry ${ }^{34}$ (or polariton chemistry), ${ }^{3,4,23-32}$ since V-SC or V-USC can have an obvious influence on the chemical reactivity in bond-cleavage ${ }^{11,18}$ and solvolysis reactions. ${ }^{17,19}$ Impressively, for such reaction systems under V-SC or V-USC, diametrically opposed effects on reaction rates have been observed: deceleration ${ }^{11,18}$ and acceleration. ${ }^{16,17,19}$ The latter rate accelerating action has been called vacuum-field catalysis (or cavity catalysis) ${ }^{16,17,19}$ because V-SC or V-USC with the FP cavity lowers the reaction barrier (Fig. 2(b)). In practice, it has been reported that some vacuumfield catalysts can give rise to a significantly large increase in the reaction rate constant: ${ }^{17,19}$ e.g., $\sim 10$ times the rate for solvolysis (ethyl acetate) of para-nitrophenyl acetate, ${ }^{19} \sim 10^{2}$ times the rate for hydrolysis $\left(\mathrm{H}_{2} \mathrm{O}\right)$ of cyanate ions $\left(\mathrm{OCN}^{-}\right),{ }^{17}$ and $\sim 10^{4}$ times the rate for hydrolysis $\left(\mathrm{H}_{2} \mathrm{O}\right)$ of ammonia borane $\left(\mathrm{NH}_{3} \mathrm{BH}_{3}\right){ }^{17}$ Nevertheless, no kinetic model for V-SC and V-USC has yet succeeded in accounting for such large reaction rate enhancements. ${ }^{27-30}$ Considering that vacuum-field catalysis could have a potential impact on science and technology, a new kinetic model is desired to clarify the mechanism of vacuumfield catalysis and to predict its catalytic action properly.

The purpose of this paper is to provide a reaction kinetic model under V-SC or V-USC, thereby trying to give a simple but comprehensive explanation for all the reported experimental results of vacuum-field-catalyzed reactions. First, we explain vibrational light-matter coupling and consider how vacuum-field catalysis works from the viewpoint of standard physical chemistry, wherein we hypothesize that vibrational light-matter coupling may reshape a reaction potential involved in the vibropolaritons of reactants and thereby modify the reaction barrier height. Second, we show how to introduce such a bold hypothesis into the Arrhenius and Eyring-Polanyi equations, thereby obtaining two analytical formulas as a function of the coupling ratio $\Omega_{\mathrm{R}} / 2 \omega_{0}$. Third, we estimate the potential performance of vacuum-field catalysis by numerical calculations using the obtained formulas and compare our calculations with reported observations of vacuum-field-catalyzed reactions. 


\section{Theory}

\subsection{Vibrational light-matter coupling}

Figure 1 represents energy diagrams of vibrational lightmatter coupling. For simplicity, vibrational potentials are drawn as a quadratic curve under the harmonic oscillator approximation. If the optical energy of a cavity $\hbar \omega_{\mathrm{c}}$ ( $\hbar$ : reduced Planck constant) is exactly tuned to the vibrational energy of given molecules $\hbar \omega_{0}$, that is, $\hbar \omega_{\mathrm{c}}=\hbar \omega_{0}$, Rabi splitting occurs through hybridizing the molecular vibration and IR vacuum field. As a result, a pair of Rabi-split vibro-polaritonic states is formed as shown in Fig. 1(b). Such a pair of vibro-polaritons is often denoted as a lightmatter hybrid ${ }^{1-4}$ and composed of the upper vibro-polariton $P_{+}$ with an energy of $\hbar \omega+$ and the lower vibro-polariton $P_{-}$with an energy of $\hbar \omega_{-}$. The difference in energy between $P_{+}$and $P_{+}$is called the Rabi splitting energy $\hbar \Omega_{\mathrm{R}}$ and described as follows: ${ }^{1,2}$

$$
\hbar \Omega_{\mathrm{R}}=2 \sqrt{N} E d=2 \sqrt{N} \sqrt{\frac{\hbar \omega_{0}}{2 \varepsilon_{0} V}} \mathrm{~d} \sqrt{n_{\mathrm{ph}}+1}
$$

where $N$ is the number of molecules coupled to the cavity mode, $E$ is the amplitude of the electric field of light, $d$ is the transition dipole moment of the molecules, $n_{\mathrm{ph}}$ is the number of photons populating the cavity mode, $\varepsilon_{0}$ is the dielectric constant of vacuum and $V$ is the mode volume. The most striking aspect of Eq. 1 is that even if $n_{\mathrm{ph}}=0, \hbar \Omega_{\mathrm{R}}$ may have a finite value due to the quantum vacuum fluctuation of electromagnetic field. ${ }^{1-4}$ The presence of such residual $\hbar \Omega_{\mathrm{R}}$ is crucial for vacuum-field catalysis because it ensures that vacuum-field catalysis works as a reaction booster even without any photons. Moreover, in common with conventional catalysts, vacuum-field catalysis requires no additional energy to function as a catalyst.
The absolute value of $\hbar \Omega_{R}$ varies from system to system. For comparison of such systems with different energy scales, the coupling ratio (or coupling strength) is defined as the ratio of half $\Omega_{\mathrm{R}}$ to $\omega_{0}$, i.e., $\Omega_{\mathrm{R}} / 2 \omega_{0}{ }^{35,36}$ The boundary between the strong and ultra strong coupling regimes is most often set at $\Omega_{\mathrm{R}} / 2 \omega_{0}=$ $0.1,{ }^{3,4,35-38}$ whereas if $\Omega_{\mathrm{R}} / 2 \omega_{0} \geq 1$, the regime of the light-matter interaction is called deep strong coupling. ${ }^{39,40}$ However, no vibrational deep strong coupling has been found so far: the largest $\Omega_{\mathrm{R}} / 2 \omega_{0}$ was $\sim 0.13$ observed for the $\mathrm{OH}$ stretch of ice. ${ }^{33}$

The ratio of vibro-polariton energy $\hbar \omega_{ \pm}$to the original vibrational energy $\hbar \omega_{0}$ is expressed as a function of $\Omega_{\mathrm{R}} / 2 \omega_{0}$ as follows: ${ }^{35,41}$

$$
\frac{\omega_{ \pm}}{\omega_{0}}=\left(1 \pm \frac{1}{2} \frac{\Omega_{\mathrm{R}}}{\omega_{0}}\right)
$$

$\frac{\omega_{ \pm}}{\omega_{0}}=\sqrt{1+\left(\frac{1}{2} \frac{\Omega_{\mathrm{R}}}{\omega_{0}}\right)^{2}} \pm \frac{1}{2} \frac{\Omega_{\mathrm{R}}}{\omega_{0}}$

Both Eqs. 2 and 3 are derived from eigenvalues of Hopfield's Hamiltonian: ${ }^{3,35}$ on the one hand, the former may be valid only if $\Omega_{\mathrm{R}} / 2 \omega_{0} \ll 1$, wherein the anti-resonance term of the lightmatter coupling is neglected in the Hamiltonian (rotational wave approximation). On the other hand, the latter may be valid even if $\Omega_{\mathrm{R}} / 2 \omega_{0}$ is nonnegligible compared to unity $\left(\Omega_{\mathrm{R}} / 2 \omega_{0} \approx 1\right)$, wherein the anti-resonance term is included. ${ }^{35} \mathrm{Eq} .3$ is thus more generic than Eq. 2 in that the former is valid for multiple regimes from weak to deep strong coupling. We henceforth utilize Eq. 3 for the following discussion.

\begin{tabular}{|c|c|c|c|c|c|c|c|c|}
\hline molecule & $\begin{array}{l}\text { vibrational } \\
\text { mode }\end{array}$ & ${ }^{[\mathrm{d}]} d[\mathrm{D}]$ & $\omega_{0}\left[\mathrm{~cm}^{-1}\right]$ & $\Omega_{\mathrm{R}}\left[\mathrm{cm}^{-1}\right]$ & $\Omega_{\mathrm{R}} / 2 \omega_{0}$ & $\begin{array}{c}E \times 10^{-4} \\
{\left[\mathrm{MV} \cdot \mathrm{cm}^{-1}\right]}\end{array}$ & $N \times 10^{11}$ & $\begin{array}{c}\text { coupling } \\
\text { regime }\end{array}$ \\
\hline${ }^{[\mathrm{a}]}$ acetonitrile & $\mathrm{C} \equiv \mathrm{N}$ str. & 0.061 & 2255 & 41 & 0.0091 & 0.83 & 0.59 & $\mathrm{~V}-\mathrm{SC}$ \\
\hline [a] toluene & $\mathrm{C}-\mathrm{H}$ str. & 0.14 & 3041 & 87 & 0.014 & 1.7 & 0.11 & V-SC \\
\hline [b] poly(vinyl acetate) & $\mathrm{C}=\mathrm{O}$ str. & 0.24 & 1740 & 167 & 0.048 & 0.60 & 1.2 & V-SC \\
\hline [a] phenyl isocyanate & $\mathrm{N}=\mathrm{C}=\mathrm{O}$ str. & 0.51 & 2272 & 308 & 0.068 & 1.0 & 0.32 & V-SC \\
\hline${ }^{\text {[a] }} \mathrm{H}_{2} \mathrm{O}$ water & $\mathrm{O}-\mathrm{H}$ str. & 0.34 & 3400 & 740 & 0.11 & 1.8 & 1.3 & V-USC \\
\hline${ }^{[\mathrm{c}]} \mathrm{Fe}(\mathrm{CO})_{5}$ & $\mathrm{C} \equiv \mathrm{O}$ str. & 0.88 & 2000 & 480 & 0.12 & 0.79 & 0.42 & V-USC \\
\hline${ }^{[\mathrm{a}]} \mathrm{H}_{2} \mathrm{O}$ ice & $\mathrm{O}-\mathrm{H}$ str. & 0.41 & 3250 & 820 & 0.13 & 1.7 & 1.3 & V-USC \\
\hline
\end{tabular}

Table 1. Examples of physical quantities for vibrational vacuum Rabi Splitting

[a] Ref. 33. [b] Refs. 5, 33. [c] Refs. 9, 33. [d] The unit is in debye (D), where $1 \mathrm{D}=3.33564 \times 10^{-30} \mathrm{C} \cdot \mathrm{m}$.

Table 1 summarizes typical numerical values of vibrational vacuum-field Rabi splitting for several compounds reported in the literature. ${ }^{5,9,17,33}$ The values of $E$ and $N$ were calculated according to Eq. 1 , assuming that $n_{\mathrm{ph}}=0$ and the mode volume is diffraction-limited as $V \approx(\lambda / n)^{3}(\lambda$ : wavelength of the vibrational mode, $n$ : refractive index of the cavity media). ${ }^{5}$ The vacuum Rabi splitting is sometimes regarded as a kind of optical (or dynamical) Stark effect, ${ }^{42,43}$ although the former occurs in the absence of any external electric fields $\left(n_{\mathrm{ph}}=0\right)$. Let us thus compare the vibrational Rabi splitting and the vibrational Stark effect $^{43-45}$. On the one hand, for the vibrational Stark effect, the observed Stark shift ranges from approximately 0.2 to $3 \mathrm{~cm}^{-1}$ under an external electrostatic field of $1 \mathrm{MV} \cdot \mathrm{cm}^{-1} \cdot{ }^{44}$ On the other hand, for vibrational Rabi splitting, a corresponding shift $\left(\sim \Omega_{R} / 2\right)$ ranges from approximately 20 to $400 \mathrm{~cm}^{-1}$ under an IR vacuum field of $\sim 10^{-4} \mathrm{MV} \cdot \mathrm{cm}^{-1}$ according to Table 1 . In other words, the vibrational Rabi splitting gives a hundred times larger frequency shifts under a ten thousandth time smaller electric field than the vibrational Stark effect. This six-digit difference originates in the square root term $\sqrt{N} \approx 10^{5}$ in Eq. 1 . Apparently, the number density $N$ empowers the vacuum-field Rabi splitting to possess a $\sqrt{N}$-multiplied collective quantity, ${ }^{47,48}$
$\sqrt{N} E\left(16 \sim 65 \mathrm{MV} \cdot \mathrm{cm}^{-1}\right)$ or $\sqrt{N} d\left(10^{4} \sim 10^{5} \mathrm{D}\right)$. It should be noted that a strong electrostatic interaction can give rise to high chemical reactivity: e.g., it is known that a Stark shift of the $\mathrm{C}=\mathrm{O}$ stretching mode reaches up to $\sim 100 \mathrm{~cm}^{-1}$ in the highly dielectric environment of a special enzyme (ketosteroid isomerase), which exerts an electrostatic field of $\sim 140 \mathrm{MV} \cdot \mathrm{cm}^{-1} \cdot{ }^{45,46}$ Under such a strong electrostatic interaction, the enzyme serves as an electricfield (or electrostatic) catalyst ${ }^{45,46,49}$ to initiate a protonrearrangement reaction $\sim 10^{5}$-fold faster than the nonenzymatic reaction with no Stark effect. Hence, it is very likely that vibrational light-matter coupling may drastically promote chemical reactions because $\Omega_{\mathrm{R}} / 2$ reaches up to $\sim 400 \mathrm{~cm}^{-1}$.

Finally, in this section, we describe the main hypothesis in this study. When transitioning from the original vibrational potential to the vibro-polaritons' potential (Fig. 1(a) to 1(b)), we hypothesize the reshape in potential under the vibrational lightmatter coupling: the steeper potential for the $P_{+}$vibro-polariton (blue quadric curve) and the shallower potential for the $P$-vibropolariton (red quadric curve). This hypothesis is deduced from the following considerations: first, according to Eqs. 2 or 3, $\omega+$ $>\omega_{0}$ and $\omega_{-}<\omega_{0}$. Second, the force constant $k_{0}$ (the original force constant) and $k_{ \pm}$(the force constant for the $P_{ \pm}$vibro- 
polaritons) are described as $k_{0}=m_{0} \omega_{0}^{2}$ and $k_{ \pm}=m_{0} \omega_{ \pm}^{2}$, respectively, where $m_{0}$ is the reduced mass of the given molecular vibration. Here, we assume that the reduced mass remains unchanged under vibrational light-matter coupling. It is thus obvious that $k_{+}>k_{0}$ and $k-<k_{0}$. Third, since the force constant is the second derivative of the vibrational potential, the inequality relations, $k_{+}>k_{0}$ and $k_{-}<k_{0}$, indicate steeper and shallower potentials than the original potential, respectively. In this view, we consider the abovementioned extremely large $\sqrt{N} E$ or $\sqrt{N} d$ as a driving force of the reshape in potential.

\subsection{Qualitative picture of vacuum-field catalysis}

Figure 2(a) displays a schematic comparison of reaction potentials with and without vibrational light-matter coupling: the original potential is drawn as a black curve, whereas lightcoupled $P_{+}$and $P_{-}$potentials, which are derived from the upper and lower vibro-polaritons, are drawn as blue and red curves, respectively. Here, we suppose that vibrational light-matter coupling is applied to a certain vibrational mode directly relevant to the bond-cleavage of the given reaction. In the same fashion as discussed before, when transitioning from the original potential to the light-coupled potentials, the force constant changes from $k_{0}$ to $k_{ \pm}$in accordance with a frequency change from $\omega_{0}$ to $\omega_{ \pm}$. We hypothesize that such changes in force constant reshape the original reaction potential, resulting in the formation of the steeper $P_{+}$potential (colored in blue) and the

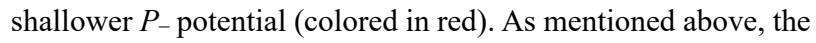
origin of the reshape in potential is due to the apparent giant IR vacuum field $\sqrt{N} E$ or the apparent giant transition dipole moment $\sqrt{N} d$. Such reshape in potential further make a difference in activation energy: the $P_{+}$potential has a high activation energy $E_{+}$, whereas the $P_{-}$potential has a low

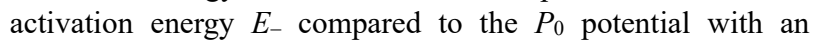
original activation energy $E_{0}$. Thus, chemical reactions under vibrational light-matter coupling proceed more slowly or more rapidly when passing through the $P_{+}$or $P_{-}$potential, respectively. In other words, $\kappa_{+}<\kappa_{0}$ and $\kappa_{-}>\kappa_{0}$, where $\kappa_{+}$is the reaction rate constant via the $P_{+}$potential, $\kappa_{0}$ is the original reaction rate constant, and $\kappa_{-}$is the reaction rate constant via the $P_{-}$potential. Since $\kappa^{+}<\kappa_{0}<\kappa_{-}$, the reaction on the $P$ - potential dominates that on the $P+$ potential. As a result, the reactants on the $P_{-}$potential act a rate enhancer.

Figure 2(b) illustrates a basic design of the vacuum-field catalyst using an FP cavity. If a certain vibrational mode involved in the bond-cleavage is coupled to IR vacuum field, a catalytic space may be spanned between a pair of mirrors of an FP cavity. The resonance can be tuned simply by adjusting the cavity length of the FP cavity (typically $1-20 \mu \mathrm{m}$ ). On the one hand, the catalytic space is half-filled with reactants that are chemically activated by IR vacuum field and follow the $P_{-}$ potential: they drive reactions faster than those on the original potential. On the other hand, the remaining half of reactants on the $P_{+}$potential have little contribution to the total reaction as explained above.

From such points of view, we may withdraw the following three characteristics of vacuum-field catalysts:

(1) The catalytic mechanism of vacuum-field catalysis entirely differs from that of conventional catalysis: the vacuumfield catalyst catalyzes a reaction by directly lowering its activation barrier without changing its reaction path, whereas the conventional catalyst catalyzes a reaction by changing its path to seek a barrier as low as possible.

(2) Vacuum-field catalysis exert a 3-dimentional catalytic action without touching with reactants, which is a unique feature unseen for conventional catalysts. Hence, vacuum-field catalysis may find new importance as such a remote catalyst.
(3) The catalytic action of vacuum-field catalysis is dependent on the cavity length and the coupling ratio $\Omega_{R} / 2 \omega_{0}$ of the vibration involved in the reaction. The rate-enhancement is a function of $\Omega_{R} / 2 \omega_{0}$ as shown below.

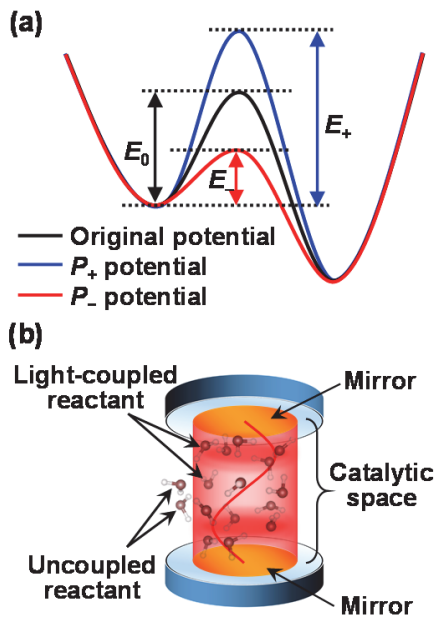

Figure 2. (a) Schematic comparison of reaction potentials with and without vibrational light-matter coupling. (b) Basic design of vacuum-field catalysis using an FP cavity.

\subsection{Formulation of reaction-rate equations for light- coupled reactions}

Let us consider a simple reaction for vacuum-field catalysis: for example, $\mathrm{AB}+\mathrm{C} \rightarrow \mathrm{A}+\mathrm{BC}$ as shown Fig. 3. If the original reaction potential $U_{0}(r)$ of the molecule A-B takes a form of the Morse potential, $U_{0}(r)$ and the $n$th derivative of $U_{0}(r)$, i.e., $U_{0}^{(\mathrm{n})}(r)$ are respectively described as ${ }^{52}$

$U_{0}(r)=D_{0}\left[\exp \left\{-2 \alpha_{0}\left(r-r_{\mathrm{e} 0}\right)\right\}-2 \exp \left\{-\alpha_{0}\left(r-r_{\mathrm{e} 0}\right)\right\}\right]$

$U_{0}^{(n)}(r)=$

$(-\alpha)^{n} D_{0}\left[2^{n} \exp \left\{-2 \alpha_{0}\left(r-r_{\mathrm{e} 0}\right)\right\}-2 \exp \left\{-\alpha_{0}\left(r-r_{\mathrm{e} 0}\right)\right\}\right]$

where $D_{0}$ is the original dissociation energy of $\mathrm{A}-\mathrm{B}, \alpha_{0}$ is the parameter related to the width of the original Morse potential, and $r_{\mathrm{e} 0}$ is the original interatomic distance of $\mathrm{A}-\mathrm{B}$ at equilibrium. Here, $\alpha_{0}$ is expressed as $\alpha_{0}=\sqrt{k_{0} / 2 D_{0}}$, where $k_{0}$ is the original force constant of the A-B stretching mode and is set up as $k_{0}=$ $U_{0}^{(2)}\left(r_{\mathrm{e} 0}\right)=\alpha_{0}^{2} D_{0}$. Furthermore, $k_{0}$ is defined as $k_{0}=m \omega_{0}^{2}$, where $m$ is the reduced mass of $\mathrm{A}-\mathrm{B}$, and $\omega_{0}$ is the original molecular frequency of the A-B stretching mode, hence, $\alpha_{0}^{2} D_{0}=m \omega_{0}^{2}$. Taking into account that $U_{0}^{(n)}\left(r_{\mathrm{e} 0}\right)=(-\alpha)^{n} D_{0}\left(2^{n}-2\right) / n$ !he original activation energy $E_{0}$ may be given by a Taylor expansion of Eq. 4 about a point of $r_{\mathrm{e} 0}$ as

$E_{0}=U_{0}\left(a_{0}\right)-U_{0}\left(r_{\mathrm{e} 0}\right)=D_{0} \sum_{n=1}^{\infty} \frac{\alpha^{n}\left(2^{n}-2\right)}{n !}\left(r_{e 0}-a_{0}\right)^{n}$

where $a_{0}$ is the original coordinate at the transition state of the original reaction. Note that the series in Eq. 6 converges for all $a_{0}$ since the Cauchy root test $\mathrm{t}^{52,53}$ gives $\lim _{n \rightarrow \infty}\left\{\frac{\alpha^{n}\left(2^{n}-2\right)}{n !}\right\}^{\frac{1}{n}}=0<1$.

Thus, Eq. 6 can be simplified by the harmonic oscillator approximation as

$$
\begin{aligned}
E_{0} & \approx \alpha_{0}{ }^{2} D_{0}\left(a_{0}-r_{e 0}\right)^{2} \\
& =\frac{1}{2} k_{0}\left(a_{0}-r_{e 0}\right)^{2}=\frac{1}{2} m \omega_{0}{ }^{2}\left(a_{0}-r_{e 0}\right)^{2}
\end{aligned}
$$

If the same manner is applied to the light-coupled reaction, the activation energy for the $P_{ \pm}$potentials $E_{ \pm}$may be written as

$$
\begin{aligned}
E_{ \pm} & \approx \alpha_{ \pm}{ }^{2} D_{ \pm}\left(a_{ \pm}-r_{e \pm}\right)^{2} \\
& =\frac{1}{2} k_{ \pm}\left(a_{ \pm}-r_{e \pm}\right)^{2}=\frac{1}{2} m \omega_{ \pm}^{2}\left(a_{ \pm}-r_{e \pm}\right)^{2}
\end{aligned}
$$


where $D_{ \pm}, a_{ \pm}, r_{\mathrm{e} \pm}$, and $k_{ \pm}$are, respectively, the dissociation energy of $\mathrm{A}-\mathrm{B}$, the coordinate at the transition state, the interatomic distance of $\mathrm{A}-\mathrm{B}$ at equilibrium, and the force constant of the A$B$ stretching mode, all of which are defined under vibrational light-matter coupling. We assume here that the reduced mass $m$ remains intact regardless of the absence and presence of vibrational light-matter coupling.

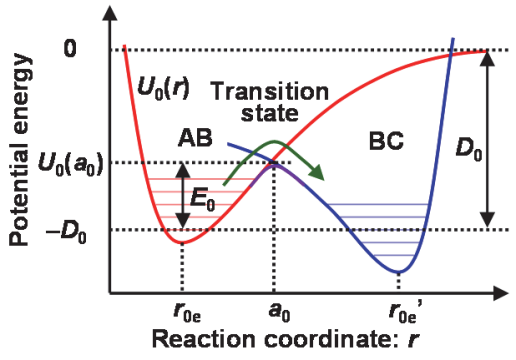

Figure 3. Original reaction potential $U_{0}(r)$ of a simple dissociation, $\mathrm{AB}+\mathrm{C} \rightarrow \mathrm{A}+\mathrm{BC}$, as a function of the reaction coordinate $r$.

If dividing Eq. 8 by Eq. 7, then introducing Eq. 3, the relative activation energy $E_{ \pm} / E_{0}$ may be obtained as follows: ${ }^{54}$

$$
\begin{aligned}
\frac{E_{ \pm}}{E_{0}} & \approx \frac{\alpha_{ \pm}^{2} D_{ \pm}}{\alpha_{0}^{2} D_{0}}\left(\frac{a_{ \pm}-r_{\mathrm{e} \pm}}{a_{0}-r_{\mathrm{e} 0}}\right)^{2} \approx \frac{\alpha_{ \pm}{ }^{2} D_{ \pm}}{\alpha_{0}^{2} D_{0}}=\left(\frac{k_{ \pm}}{k_{0}}\right)^{2}=\left(\frac{\omega_{ \pm}}{\omega_{0}}\right)^{2} \\
& =\left(\sqrt{1+\left(\frac{1}{2} \frac{\Omega_{\mathrm{R}}}{\omega_{0}}\right)^{2}} \pm \frac{1}{2} \frac{\Omega_{\mathrm{R}}}{\omega_{0}}\right)^{2}
\end{aligned}
$$

wherein we assume that $\left(a_{ \pm}-r_{\mathrm{e} \pm}\right) /\left(a_{0}-r_{\mathrm{e} 0}\right) \approx 1$ because the coordinate of the transition state point is moved nearly parallel to that of the equilibrium point when transitioning from the original potential to the $P_{ \pm}$potentials. Now we can relate the thermodynamic quantity $E_{ \pm} / E_{0}$ to the quantum-electrodynamic quantity $\Omega_{\mathrm{R}} / 2 \omega_{0}$. Notice that when developing Eq. 9, we presuppose the reshape in potential.

We next incorporate $\Omega_{\mathrm{R}} / 2 \omega_{0}$ into the Arrhenius and Eyring-Polanyi equations using Eq. 9, thereby obtaining the relative reaction rate constant $\kappa_{ \pm} / \kappa_{0}$ as a function of $\Omega_{\mathrm{R}} / 2 \omega_{0}$. In general, the Arrhenius equation is regarded as an empirical relation between $\kappa_{0}$ and $E_{0},{ }^{53,55}$ whereas the Eyring-Polanyi equation is deductively derived from transition state theory. ${ }^{55,56}$ For the original reaction, each of them is respectively described as follows: $:^{53,55,56}$

$\kappa_{0}=A_{0} \exp \left(-\frac{E_{0}}{k_{\mathrm{B}} T}\right)$

$\kappa_{0}=\left(\frac{a_{0}}{r_{\mathrm{e} 0}}\right)^{2} \frac{\omega_{0}}{2 \pi} \exp \left(-\frac{E_{0}}{k_{\mathrm{B}} T}\right)$

where $A_{0}$ is the pre-exponential factor (or frequency of collisions) for the original reaction, $T$ is the absolute temperature in kelvin and $k_{\mathrm{B}}$ is the Boltzmann constant. For Eq. 11, we selected an expression for the simplest dissociation reaction among many types of Eyring-Polanyi equations. ${ }^{55}$ Similarly, for the light-coupled reaction, the following Arrhenius and EyringPolanyi equations may be respectively expressed as

$\kappa_{ \pm}=A_{ \pm} \exp \left(-\frac{E_{ \pm}}{k_{\mathrm{B}} T}\right)$

$\kappa_{ \pm}=\left(\frac{a_{ \pm}}{r_{\mathrm{e} \pm}}\right)^{2} \frac{\omega_{ \pm}}{2 \pi} \exp \left(-\frac{E_{ \pm}}{k_{\mathrm{B}} T}\right)$

where $A_{ \pm}$is the pre-exponential factor for the light-coupled reaction. If dividing Eqs. 12 and 13 respectively by Eqs. 10 and 11, followed by substituting Eqs. 3 and 9, the relative reaction rate constant $\kappa_{ \pm} / \kappa_{0}$ may be respectively obtained as follows: ${ }^{57,58}$

$$
\begin{aligned}
\frac{\kappa_{ \pm}}{\kappa_{0}} & =\frac{A_{ \pm}}{A_{0}} \exp \left[\left(-\frac{E_{0}}{k_{\mathrm{B}} T}\right)\left\{\left(\frac{E_{ \pm}}{E_{0}}\right)-1\right\}\right] \\
& \approx \exp \left[\left(-\frac{E_{0}}{k_{\mathrm{B}} T}\right)\left\{\left(\sqrt{1+\left(\frac{1}{2} \frac{\Omega_{\mathrm{R}}}{\omega_{0}}\right)^{2}} \pm \frac{1}{2} \frac{\Omega_{\mathrm{R}}}{\omega_{0}}\right)^{2}-1\right\}\right] \\
\frac{\kappa_{ \pm}}{\kappa_{0}} & =\frac{\left(\frac{a_{ \pm}}{r_{\mathrm{e} \pm}}\right)^{2}}{\left(\frac{a_{0}}{r_{\mathrm{e} 0}}\right)^{2}} \frac{\omega_{ \pm}}{\omega_{0}} \exp \left[\left(-\frac{E_{0}}{k_{\mathrm{B}} T}\right)\left\{\left(\frac{E_{ \pm}}{E_{0}}\right)-1\right\}\right] \\
& \approx\left\{\sqrt{1+\left(\frac{1}{2} \frac{\Omega_{\mathrm{R}}}{\omega_{0}}\right)^{2}} \pm \frac{1}{2} \frac{\Omega_{\mathrm{R}}}{\omega_{0}}\right\} \\
& \times \exp \left[\left(-\frac{E_{0}}{k_{\mathrm{B}} T}\right)\left\{\left(\sqrt{1+\left(\frac{1}{2} \frac{\Omega_{\mathrm{R}}}{\omega_{0}}\right)^{2}} \pm \frac{1}{2} \frac{\Omega_{\mathrm{R}}}{\omega_{0}}\right)^{2}-1\right\}\right]
\end{aligned}
$$

In the Arrhenius-type Eq. 14, we assumed $A_{ \pm} / A_{0} \approx 1$ because there is no or a negligible difference in velocities, collision cross sections, and concentrations of reactant molecules between the original and light-coupled reactions. In addition, in the EyringPolanyi-type Eq. 15 , we assumed $\left(a_{ \pm} / r_{\mathrm{e} \pm}\right) /\left(a_{0} / r_{\mathrm{e} 0}\right) \approx 1$ because the coordinate of the transition state point is moved nearly parallel to that of the equilibrium point when transitioning from the original potential to the $P_{ \pm}$potentials. Finally, the reactionkinetic quantity $\kappa_{ \pm} / \kappa_{0}$ can be expressed as a function of the quantum-electrodynamic quantity $\Omega_{\mathrm{R}} / 2 \omega_{0}$. Both Eqs. 14 and 15 are valid for the multiple regimes from weak to deep strong coupling because they are derived from Eq. 3, which is valid for all the coupling regimes. In the above discussion, we used the Morse potential as a reaction potential. Instead, if using another molecular potential, like the Lenard-Jones potential, the exact same equations including Eqs. 9, 14, and 15 may be acquired as long as the following conditions hold: the potential is Taylorexpandable, $a_{ \pm}-r_{\mathrm{e} \pm} \approx a_{0}-r_{\mathrm{e} 0}$, and $a_{ \pm} / r_{\mathrm{e} \pm} \approx a_{0} / r_{\mathrm{e} 0}$.

Next, we compare $\kappa_{+} / \kappa_{0}$ and $\kappa_{-} / \kappa_{0}$ in Eqs. 14 and 15 . Notice that $\kappa_{+} / \kappa_{0}$ and $\kappa_{-} / \kappa_{0}$ correspond to the decelerated and accelerated reactions because $\kappa_{+} / \kappa_{0}<1$ and $\kappa_{-} / \kappa_{0}>1$, respectively. Let us consider $\kappa_{+} / \kappa_{-}$, which may be obtained if using Eq. 14 as

$\frac{\kappa_{+}}{\kappa_{-}}=\exp \left\{4\left(-\frac{E_{0}}{k_{\mathrm{B}} T}\right) \sqrt{1+\left(\frac{1}{2} \frac{\Omega_{\mathrm{R}}}{\omega_{0}}\right)^{2}}\left(\frac{1}{2} \frac{\Omega_{\mathrm{R}}}{\omega_{0}}\right)\right\}$

On the one hand, if assuming a typical reaction under $\mathrm{V}-\mathrm{SC}$, for example, $E_{0}=0.5 \mathrm{eV}, T=300 \mathrm{~K}$, and $\Omega_{\mathrm{R}} / 2 \omega_{0}=0.05$, Eq. 16 gives $\kappa_{+} / \kappa_{-} \approx 0.02$, indicating that compared to $\kappa_{-}, \kappa_{+}$contributes little to total reaction rate constant as qualitatively discussed before. More generally, if assuming that the reactants lie on the $P_{+}$and $P_{-}$potentials with equal probability, the total reaction rate constant under V-SC or V-USC $\kappa_{\text {total }}$ may be expressed as

$\kappa_{\text {total }}=\frac{1}{2}\left(\kappa_{-}+\kappa_{+}\right)=\frac{1}{2} \kappa_{-}\left(1+\frac{\kappa_{+}}{\kappa_{-}}\right) \approx \frac{1}{2} \kappa_{-}$

Eq. 17 teaches us that $\kappa$ - may determine $\kappa_{\text {total }}$ unless $\Omega_{\mathrm{R}} / 2 \omega_{0}$ is too small. On the other hand, if assuming that the access routes to the $P_{+}$and $P_{-}$potentials are not equiprobable, the situation may change:, e.g., if the access to the $P$ - potential occurs with zero probability, $\kappa+$ may inevitably determine $\kappa$ total. We suspect that such situations might happen to the decelerated reactions under $\mathrm{V}-\mathrm{SC},{ }^{11,18}$ but we have no particular idea how the reaction path on the $P_{-}$potential could be closed. Since our aim is to explain the catalytic action of vacuum-field catalysis, we hereafter focus on $\kappa-/ \kappa_{0}$ for accelerated reactions and evaluate the performance of vacuum-field catalysis based on Eqs. 14 and 15 with $\kappa_{-} / \kappa_{0}$. 


\section{Results and Discussion \\ 3.1. Performance of vacuum-field catalysis predicted by the reaction kinetic model in this study}

Figure 4 depicts the comparison of $\kappa-/ \kappa_{0}$ versus $\Omega_{\mathrm{R}} / 2 \omega_{0}$ with various $E_{0}$ when $T=300 \mathrm{~K}$ (room temperature). The values of $\kappa / \kappa_{0}$ were numerically calculated using Eqs. 14 (Arrheniustype) and 15 (Eyring-Polanyi-type). The characteristics of Fig. 4 are summarized as follows:

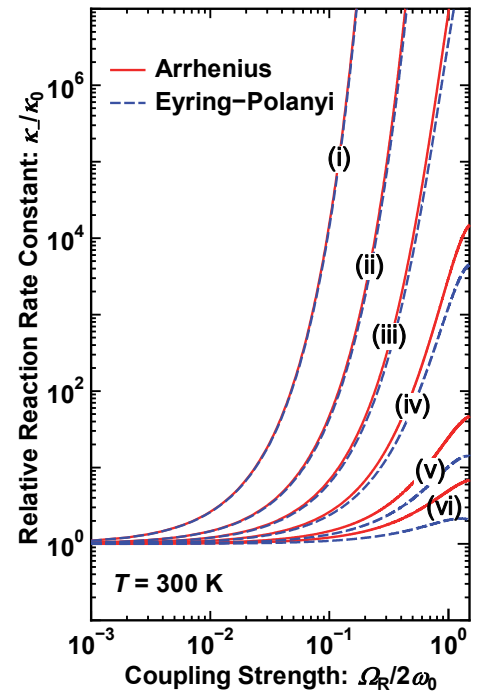

Figure 4. Comparison of relative reaction rate constant $\kappa-/ \kappa_{0}$ versus coupling ratio $\Omega_{\mathrm{R}} / 2 \omega_{0}$ with various activation energies $E_{0}$ : (i) $E_{0}=2.50 \mathrm{eV}$, (ii) $E_{0}=1.00 \mathrm{eV}$, (iii) $E_{0}=0.50 \mathrm{eV}$, (iv) $E_{0}=$ $0.25 \mathrm{eV}$, (v) $E_{0}=0.10 \mathrm{eV}$, (vi) $E_{0}=0.05 \mathrm{eV}$; red solid curve: Arrhenius type (Eq. 14), blue dashed curve: Eyring-Polanyi type (Eq. 15).

(1) In all cases in both the Arrhenius-type and EyringPolanyi-type, $\kappa / \kappa_{0}$ is always larger than unity for any $\Omega_{\mathrm{R}} / 2 \omega_{0}$ and $E_{0}$, manifesting that the vibrational light-matter coupling can promote any reactions. In other words, vacuum-field catalysts functions as a universal rate-accelerator. Nonetheless, we emphasize that there are several limitations to vacuum-field catalysis as explained in the following (2) - (4).

(2) In all cases, $\kappa_{-} / \kappa_{0}$ increases exponentially with an increase of $\Omega_{R} / 2 \omega_{0}$. This trend means that $\Omega_{R} / 2 \omega_{0}$ is a decisive factor to define the catalytic action of vacuum-field catalysis as mentioned earlier. On the one hand, if taking a close look at the case of $E_{0}=0.5 \mathrm{eV}$, the value of which is typical as an activation energy, the acceleration in rate is not more than 50 under V-SC. On the other hand, for example, under the V-USC with $\Omega_{\mathrm{R}} / 2 \omega_{0}$ $=0.3, \kappa^{\prime} / \kappa_{0}$ increases above $10^{4}$. Thus, if a drastic acceleration is desired, the vacuum-field catalyst should be used under V-USC.

(3) For both the Arrhenius-type and Eyring-Polanyi-type, if $\Omega_{\mathrm{R}} / 2 \omega_{0}$ is fixed, $\kappa / \kappa_{0}$ may increase as $E_{0}$ increases. This trend is explained in terms of the absolute reduction in activation energy $E_{0}-E_{-}$. This value is always larger for the vacuum-fieldcatalyzed reaction with high $E_{0}$ than for that with low $E_{0}$, even though both the high- $E_{0}$ and low- $E_{0}$ reactions receive the same relative reduction in activation energy $E_{-} / E_{0}$ according to Eq. 9. From the reverse point of view, vacuum-field catalysis may be almost useless if the absolute value of $E_{0}$ is too small: if $E_{0}$ is near and below the thermal energy of the reaction environment (e.g., $\sim 0.025 \mathrm{eV}$ at $300 \mathrm{~K}$ ), $\kappa_{-} / \kappa_{0}<2$ according to Eqs. 14 and 15 .

(4) Since $\Omega_{R} / 2 \omega_{0}$ is proportional to the product of three quantities $\sqrt{N}$ (or $\sqrt{N / V}$ ), $d$, and $E$ according to Eq. 1, Eqs. 14 and 15 indicate that they should be made as large as possible for gaining large rate enhancement. On the one hand, although the confinement of $V$ brings about an increase of $E$, it causes a decrease in $\sqrt{N / V}$ at the same time. Namely, the changes in $E$ and $\sqrt{N / V}$ get cancelled out in total, implying that for the purpose of making $\Omega_{\mathrm{R}} / 2 \omega_{0}$ larger, it is ineffective to improve the quality of an FP cavity. On the other hand, molecules with large $d$ are few in number. Moreover, it is rather hard to find a suitable combination of $d$ and $\sqrt{N / V}$ in molecular liquids: ${ }^{33} \mathrm{~V}$-USC has been realized solely for $\mathrm{Fe}(\mathrm{CO}) 5,{ }^{9} \mathrm{H}_{2} \mathrm{O},{ }^{17,33}$ and $\mathrm{D}_{2} \mathrm{O}^{17,33}$ among pure (neat) liquids. Furthermore, $\sqrt{N / V}$ must be treated as a variable quantity for solution reactions, where light-coupled reactants may be diluted with a reaction solvent: $\sqrt{N / V}$ decreases as the reaction proceeds, resulting in a gradual decrease in $\Omega_{\mathrm{R}} / 2 \omega_{0}$. As a consequence, $\kappa_{-} / \kappa_{0}$ eventually converges to unity according Eq. 14 or 15 . A possible way to avoid such a depletion effect in $\Omega_{\mathrm{R}} / 2 \omega_{0}$ is to utilize light-coupled molecules that act both a reactant and a solvent, like water for hydrolysis ${ }^{17}$ and ethyl acetate for solvolysis ${ }^{19}$. Water is especially invaluable for vacuum-field catalysis because it has the largest $\Omega_{\mathrm{R}} / 2 \omega_{0}$ among molecular liquids ${ }^{17,33}$ and engages in diverse reaction systems, including biological and environmental systems. Because of the intrinsically dilute nature of gases, vacuum-field catalysis is unsuitable for gaseous reactions.

(5) Although the Arrhenius-type and Eyring-Polanyi-type equations behave very similarly, the former always gives larger $\kappa_{-} / \kappa_{0}$ than the latter does at the same $E_{0}$ and $\Omega_{\mathrm{R}} / 2 \omega_{0}$. The difference between them becomes manifested when $E_{0} \lesssim 0.25$ $\mathrm{eV}$. Such differences stem from the presence of the preexponential term of Eq. 15, $\sqrt{1+\left(\Omega_{R} / 2 \omega_{0}\right)^{2}}-\Omega_{R} / 2 \omega_{0}$. For example, when $\Omega_{\mathrm{R}} / 2 \omega_{0}=0.01,0.1$, and 1 , the damping ratio of the Eyring-Polanyi type to the Arrhenius type is determined as approximately $0.01,0.1$, and 0.4 , respectively. Considering this trend, as long as vacuum-field-catalyzed reactions undergo V-SC $\left(\Omega_{\mathrm{R}} / 2 \omega_{0}<0.1\right)$, there is little difference between values of $\kappa / \kappa_{0}$ predicted by Eqs. 14 (Arrhenius-type) and 15 (Eyring-Polanyitype).

\subsection{Comparison between the kinetic-model calculations and the experimental observations}

Figure $5(\mathrm{a})$ represents $\ln \left(\kappa / \kappa_{0}\right) /\left(E_{0} / k_{\mathrm{B}} T\right)$ versus $\Omega_{\mathrm{R}} / 2 \omega_{0}$, where $\ln \left(\kappa / / \kappa_{0}\right) /\left(E_{0} / k_{\mathrm{B}} T\right)$ denotes the natural logarithm of $\kappa / / \kappa_{0}$ normalized by $E_{0} / k_{\mathrm{B}} T$ and can be obtained by taking the logarithm of both sides of Eq. 14 as follows:

$\frac{\ln \left(\frac{\kappa_{-}}{\kappa_{0}}\right)}{\left(\frac{E_{0}}{k_{\mathrm{B}} T}\right)}=1-\left(\sqrt{1+\left(\frac{1}{2} \frac{\Omega_{\mathrm{R}}}{\omega_{0}}\right)^{2}}-\frac{1}{2} \frac{\Omega_{\mathrm{R}}}{\omega_{0}}\right)^{2}$

Eq. 18 manifests that $\ln \left(\kappa_{+} / \kappa_{-}\right) /\left(E_{0} / k_{\mathrm{B}} T\right)$ depends only on $\Omega_{\mathrm{R}} / 2 \omega_{0}$, thereby enabling us to simultaneously compare various vacuumfield-catalyzed reactions with different values of $\kappa_{-} / \kappa_{0}$ and $E_{0}$.

In Fig. 5(a), the solid curve is theoretically drawn on the basis of Eq.18, whereas the experimental points are plotted as denoted by (i) to (vi). The experimental data were taken from Refs. 17 and 19, in which a detailed description of vacuum-fieldcatalyzed reactions are found. The key parameters used in Fig. 5 are summarized in Table 2. In brief, (i) and (ii) are the addition reactions of phenyl isocyanate (Ph-NCO) with alcohols (methanol $(\mathrm{MeOH})$ and 2-propanol (iPrOH), respectively) when the $\mathrm{N}=\mathrm{C}=\mathrm{O}$ stretching mode is light-coupled under $\mathrm{V}$-SC, (iii) is a series of solvolyses (ethyl acetate (EtOAc)) of paranitrophenyl acetate $\left(\mathrm{O}_{2} \mathrm{~N}-\mathrm{Ph}-\mathrm{COOCH}_{3}\right)$ with various $\Omega_{\mathrm{R}} / 2 \omega_{0}$ when the $\mathrm{C}=\mathrm{O}$ stretching mode is light-coupled under $\mathrm{V}$-SC, (iv) and $(v)$ are a series of deutrolyses/hydrolyses $\left(\mathrm{D}_{2} \mathrm{O} / \mathrm{H}_{2} \mathrm{O}\right)$ of $\mathrm{OCN}^{-}$ions with various $\Omega_{\mathrm{R}} / 2 \omega_{0}$ when the $\mathrm{O}-\mathrm{D}(\mathrm{O}-\mathrm{H})$ stretching mode is light-coupled under V-SC to V-USC, and (vi) is a hydrolysis $\left(\mathrm{H}_{2} \mathrm{O}\right)$ of $\mathrm{NH}_{3} \mathrm{BH}_{3}$ when the $\mathrm{O}-\mathrm{H}$ stretching mode is 
light-coupled under V-USC. In all cases, the vibrational mode directly relevant to the key bond-cleavage was chosen for the vibrational light-matter coupling. Note that for (ii) to (v), since the light-coupled molecules serve both as a reactant and a solvent, it is possible to maintain high $\Omega_{\mathrm{R}} / 2 \omega_{0}$ during the reaction. Although some experimental points deviate from the theoretical curve, taken as a whole, Eq. 18 is capable of properly reproducing the observed dependence of $\ln \left(\kappa^{\prime} / \kappa_{0}\right) /\left(E_{0} / k_{\mathrm{B}} T\right)$ on $\Omega_{\mathrm{R}} / 2 \omega_{0}$. In particular, two series of experimental points in (iv) and (v) are rather well fit to the theoretical curve.

Figure 5(b) compares the calculated and observed $\kappa_{-} / \kappa_{0}$, thereby enabling us to verify our model more straightforwardly. The observed values of $\kappa / \kappa_{0}$ are likewise taken from the literature ${ }^{17,19}$, while Eq. 15 (Eyring-Polanyi-type) provides the calculated values of $\kappa / / \kappa 0$. Notably, the observed values of $\kappa-/ \kappa_{0}$ are in a good agreement with the calculated values in a wide range from $\sim 10^{0}$ to $\sim 10^{4}$, although the observed data are lacking around $\sim 10^{3}$. More quantitatively, the logarithmic conversion of both the calculated and observed values of $\kappa_{-} / \kappa_{0}$ gives a positive correlation coefficient of 0.97 , implying that there is a strong linear correlation between the calculated and observed $\kappa / \kappa_{0}$. The same tendency with a correlation coefficient of +0.96 was seen if using Eq. 14 (Arrhenius-type). From the results, the kinetic model in this study can rather well account for the experimental vacuum-field-catalyzed reactions reported so far in that the catalytic action rises exponentially with an increase of $\Omega_{R} / 2 \omega_{0}$.

Finally, taking into account that numerous chemical reactions exist and exert diverse characteristics, the kinetic model in this study should be further evaluated by more different kinds of vacuum-field-catalyzed reactions. More fundamentally, since our kinetic model is built on a classical and phenomenological foundation of physical chemistry, some sort of quantum-electrodynamic description of kinetic models will be needed in future studies to understand vacuum-field catalysis more intrinsically from the viewpoint of first principles.
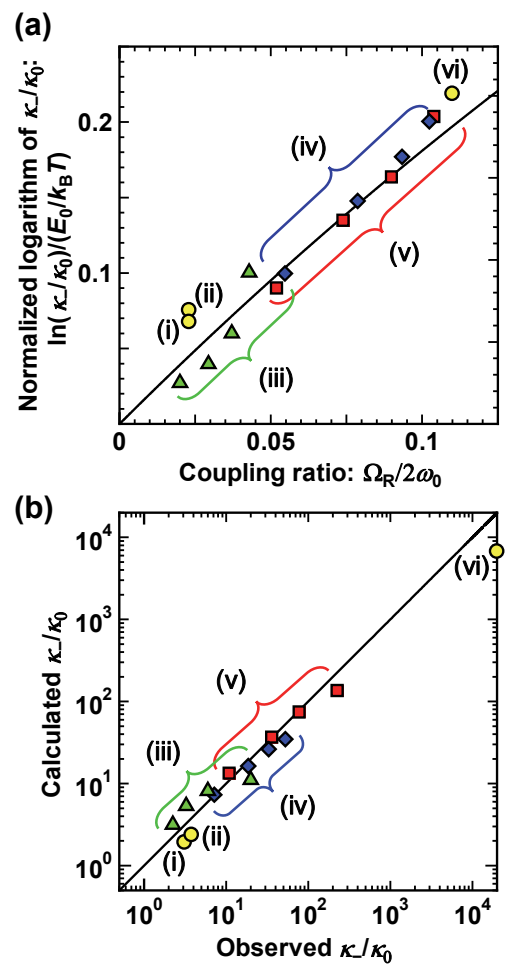

Figure 5. Comparison of calculated and observed relative reaction rate constants $\kappa / / \kappa_{0}$. (a) Normalized logarithm of $\kappa / / \kappa_{0}$ versus coupling ratio $\Omega_{\mathrm{R}} / 2 \omega_{0}$, (b) calculated $\kappa / / \kappa_{0}$ versus observed $\kappa / \kappa_{0}$ with a least-square fitted line (black solid) on a double-logarithmic scale: (i) $\mathrm{Ph}-\mathrm{NCO}+\mathrm{MeOH}$ (yellow circles), ${ }^{17}$ (ii) $\mathrm{Ph}-\mathrm{NCO}+\mathrm{iPrOH}$ (yellow circles), ${ }^{17}$ (iii) EtOAc + $\mathrm{O}_{2} \mathrm{~N}-\mathrm{Ph}-\mathrm{COOCH}_{3}$ (green triangles), ${ }^{19}$ (iv) $\mathrm{D}_{2} \mathrm{O}+\mathrm{OCN}^{-}$(blue rhombi), ${ }^{17}$ (v) $\mathrm{H}_{2} \mathrm{O}+\mathrm{OCN}^{-}$(red squares), ${ }^{17}$ (vi) $\mathrm{H}_{2} \mathrm{O}+\mathrm{NH}_{3} \mathrm{BH}_{3}$ (yellow circles). ${ }^{17}$ All the data used in Fig. 5 are summarized in Table 2.

Table 2. Summary of key parameters for various vacuum-field-catalyzed reactions.

\begin{tabular}{|c|c|c|c|c|c|c|c|}
\hline reactants & $\begin{array}{l}\text { coupled } \\
\text { vibration }\end{array}$ & $E_{0}[\mathrm{eV}]$ & $\Omega_{\mathrm{R}} / 2 \omega_{0}$ & $\begin{array}{c}\text { [c] observed } \kappa / \kappa_{0} \\
\left(2 \kappa_{\text {total }} / \kappa_{0}\right)\end{array}$ & $\begin{array}{c}\text { calculated } \kappa / \kappa_{0} \\
\text { (Arrhenius) }\end{array}$ & $\begin{array}{c}\text { calculated } \kappa / \kappa_{0} \\
\text { (Eyring-Polanyi) }\end{array}$ & $\begin{array}{l}\text { coupling } \\
\text { regime }\end{array}$ \\
\hline $\begin{array}{l}\text { [a] } \mathrm{Ph}-\mathrm{N}=\mathrm{C}=\mathrm{O}+ \\
\mathrm{MeOH}\end{array}$ & $\mathrm{N}=\mathrm{C}=\mathrm{O}$ str. & 0.39 & 0.023 & 3.12 & 1.97 & 1.93 & $\mathrm{~V}-\mathrm{SC}$ \\
\hline $\begin{array}{l}\text { [a] } \mathrm{Ph}-\mathrm{N}=\mathrm{C}=\mathrm{O}+ \\
\text { iPrOH }\end{array}$ & $\mathrm{N}=\mathrm{C}=\mathrm{O}$ str. & 0.51 & 0.023 & 3.80 & 2.43 & 2.37 & V-SC \\
\hline $\begin{array}{l}{[\mathrm{b}] \mathrm{EtOAc}+} \\
\mathrm{O}_{2} \mathrm{~N}-\mathrm{Ph}-\mathrm{COOCH}_{3}\end{array}$ & $\mathrm{C}=\mathrm{O}$ str. & 0.77 & $\begin{array}{l}0.020 \\
0.029 \\
0.037 \\
0.043\end{array}$ & $\begin{array}{l}2.2 \\
3.2 \\
6.0 \\
2.0 \times 10\end{array}$ & $\begin{array}{l}3.22 \\
5.51 \\
8.47 \\
1.16 \times 10\end{array}$ & $\begin{array}{l}3.16 \\
5.35 \\
8.16 \\
1.11 \times 10\end{array}$ & $\begin{array}{l}\text { V-SC } \\
\text { V-SC } \\
\text { V-SC } \\
\text { V-SC }\end{array}$ \\
\hline [a] $\mathrm{D}_{2} \mathrm{O}+\mathrm{OCN}^{-}$ & O-D str. & 0.51 & $\begin{array}{l}0.055 \\
0.079 \\
0.093 \\
0.102\end{array}$ & $\begin{array}{l}7.18 \\
1.86 \times 10 \\
3.30 \times 10 \\
5.26 \times 10\end{array}$ & $\begin{array}{l}7.74 \\
1.77 \times 10 \\
2.88 \times 10 \\
3.87 \times 10\end{array}$ & $\begin{array}{l}7.32 \\
1.64 \times 10 \\
2.62 \times 10 \\
3.49 \times 10\end{array}$ & $\begin{array}{r}\text { V-SC } \\
\text { V-SC } \\
\text { V-SC } \\
\text { V-USC }\end{array}$ \\
\hline [a] $\mathrm{H}_{2} \mathrm{O}+\mathrm{OCN}^{-}$ & $\mathrm{O}-\mathrm{H}$ str. & 0.69 & $\begin{array}{l}0.052 \\
0.074 \\
0.090 \\
0.104\end{array}$ & $\begin{array}{l}1.10 \times 10 \\
3.64 \times 10 \\
7.84 \times 10 \\
2.28 \times 10^{2}\end{array}$ & $\begin{array}{l}1.40 \times 10 \\
3.92 \times 10 \\
8.08 \times 10 \\
1.49 \times 10^{2}\end{array}$ & $\begin{array}{l}1.32 \times 10 \\
3.64 \times 10 \\
7.38 \times 10 \\
1.34 \times 10^{2}\end{array}$ & $\begin{array}{r}\text { V-SC } \\
\text { V-SC } \\
\text { V-SC } \\
\text { V-USC }\end{array}$ \\
\hline${ }^{[\mathrm{a}]} \mathrm{H}_{2} \mathrm{O}+\mathrm{NH}_{3} \mathrm{BH}_{3}$ & $\mathrm{O}-\mathrm{H}$ str. & 1.17 & 0.110 & $2.00 \times 10^{4}$ & $7.49 \times 10^{3}$ & $6.72 \times 10^{3}$ & V-USC \\
\hline
\end{tabular}

[a], [b] Experimental data were taken from Refs. 17 and 19, respectively. [c] The observed $\kappa / / \kappa 0$ was obtained by doubling the observed $\kappa_{\text {total }} / \kappa_{0}$ according to Eq. 17.

\section{Conclusion}

We have proposed the reaction kinetic model for vacuumfield catalysis under vibrational light-matter coupling. We have shown two kinds of analytical equations based on the Arrhenius and Eyring-Polanyi theories. We have further demonstrated that the obtained reaction-kinetic equations can properly account for the experimental results of vacuum-field-catalyzed reactions reported so far. Furthermore, we have discussed characteristics 
of vacuum-field catalysis: vacuum-field catalysts may act as a versatile rate-accelerator to promote reactions and their catalytic power is determined predominantly by the coupling ratio of Rabi frequency to molecular frequency, namely, $\Omega_{\mathrm{R}} / 2 \omega_{0}$. In particular, under the V-USC regime, the kinetic model predicts that vacuum-field catalysts can enhance the reaction rate constant by several orders of magnitude. Nevertheless, we have found from our model that if the coupling ratio is too small $\left(\Omega_{\mathrm{R}} / 2 \omega_{0} \ll 0.1\right)$ or the activation energy approaches less than the thermal energy of the reaction environment, vacuum-field catalysts may be basically ineffective as a chemical booster.

Considering the very simple structure of vacuum-field catalysts, we envision that they will find many different applications in industry. When reflecting on past views on vacuum-field catalysis, a few unusual ideas may come to mind: first, by some chance, vacuum-field catalysts might have been used for human activities without the least awareness of them, e.g., a conventional catalyst whose catalytic mechanism is enigmatic. What further intrigues us is a much more unusual hypothesis that life and nature might have already utilized the principal of vacuum-field catalysis. Otherwise, a concept of vacuum-field catalysis further allows our imaginations to run wild: vacuum-field catalysts might have played a vital role in prebiotic chemistry, that is, four billion years ago, something based on vacuum-field catalysis might have paved a short-cut from inanimate materials to life. We hope that our paper will catalyze such prospective and retrospective studies that can build a new dimension in science and technology.

\section{Acknowledgements}

H.H. acknowledges Professor Thomas W. Ebbesen for valuable discussions.

\section{References}

1. T.W. Ebbesen, Hybrid light-matter states in a molecular and material science perspective. Acc. Chem. Res. 2016, 49, 2403-2412.

2. P. Törmä, W. L. Barnes, Strong coupling between surface plasmon polaritons and emitters: a review. Rep. Prog. Phys. 2014, 78, 013901.

3. F. Kockum, A. Miranowicz, S. De Liberato, S. Savasta, F. Nori, Ultrastrong coupling between light and matter. Nat. Rev. Phys. 2019, 1, 19-40.

4. M. Hertzog, M. Wang, J. Mony, K. Börjesson, Strong light-matter interactions: a new direction within chemistry. Chem. Soc. Rev. 2019, 48, 937-961.

5. A. Shalabney, J. George, J. Hutchison, G. Pupillo, C. Genet1, T. W. Ebbesen, Coherent coupling of molecular resonators with a microcavity mode. Nat. Comm. 2015, 6, 5981.

6. A. Shalabney, J. George, H. Hiura, J. A. Hutchison, C. Genet, P. Hellwig, T. W. Ebbesen, Enhanced Raman scattering from vibro-polariton hybrid states. Angew. Chem. Int. Ed. 2015, 54, 7971-7975.

7. J. George, A. Shalabney, J. A. Hutchison, C. Genet, T. W. Ebbesen, Liquid-phase vibrational strong coupling. $J$. Phys. Chem. Lett. 2015, 6, 1027-1031.

8. J. P. Long, B. S. Simpkins, Coherent coupling between a molecular vibration and Fabry-Perot optical cavity to give hybridized states in the strong coupling limit. ACS Photonics 2015, 2, 130-136.

9. J. George, T. Chervy, A. Shalabney, E. Devaux, H. Hiura, C. Genet, T. W. Ebbesen, Multiple Rabi splittings under ultrastrong vibrational coupling. Phys. Rev. Lett. 2016, 117, 153601.

10. R. M. A. Vergauwe, J. George, T. Chervy, J. A. Hutchison,
A. Shalabney, V. Y. Torbeev, T. W. Ebbesen, Quantum strong coupling with protein vibrational modes. J. Phys. Chem. Lett. 2016, 7, 4159-4164.

11. A. Thomas, J. George, A. Shalabney, M. Dryzhakov, S. J. Varma, J. Moran, T. Chervy, X. Zhong, E. Devaux, C. Genet, J. A. Hutchison, T. W. Ebbesen, Ground-state chemical reactivity under vibrational coupling to the vacuum electromagnetic field. Angew. Chem. Int. Ed. 2016, 55, 11462-11466.

12. D. Dunkelberger, B. T. Spann, K. P. Fears, B. S. Simpkins, J. C. Owrutsky, Modified relaxation dynamics and coherent energy exchange in coupled vibration-cavity polaritons. Nat. Comm. 2016, 7, 13504.

13. M. Hertzog, P. Rudquist, J. A. Hutchison, J. George, T. W. Ebbesen, K. Börjesson, Voltage-controlled switching of strong light-matter interactions using liquid crystals. Chem. Eur. J. 2017, 23, 18166-18170.

14. B. Xiang, R. F. Ribeiro, A. D. Dunkelberger, J. Wang, Y. Lia, B. S. Simpkins, J. C. Owrutsky, J. Yuen-Zhou, W. Xiong, Two-dimensional infrared spectroscopy of vibrational polaritons. Proc. Natl. Acad. Sci. USA 2018, $115,4845-4850$.

15. V. F. Crum, S. R. Casey, J. R. Sparks, Photon-mediated hybridization of molecular vibrational states. Phys. Chem. Chem. Phys. 2018, 20, 850-857.

16. H. Hiura, H. Vibrational ultra strong coupling between light and molecules, and its application to chemistry. Bull. Solid State Phys. Appl. 2018, 24, 9-14.

17. H. Hiura, A. Shalabney, J. George, Cavity catalysis Accelerating reactions under vibrational strong coupling. 2018, ChemRxiv preprint. https://doi.org/10.26434/ chemrxiv.7234721.v3.

18. A. Thomas, L. Lethuillier-Karl, K. Nagarajan, R. M. A. Vergauwe, J. George, T. Chervy, A. Shalabney, E. Devaux, C. Genet, J. Moran, T. W. Ebbesen, Tilting a ground-state reactivity landscape by vibrational strong coupling. Science 2019, 363, 615-619.

19. J. Lather, P. Bhatt, A. Thomas, T. W, Ebbesen, J. George, Cavity catalysis by co-operative vibrational strong coupling of reactant and solvent molecules. Angew. Chem. Int. Ed. 2019, 58, 1-5.

20. J. del Pino, J. Feist, F. J. Garcia-Vidal, Quantum theory of collective strong coupling of molecular vibrations with a microcavity mode. New J. Phys. 2015, 17, 053040.

21. J. del Pino, J. Feist, F. J. Garcia-Vidal, Signatures of vibrational strong coupling in Raman scattering. J. Phys. Chem. C 2015, 119, 29132-29137.

22. J. del Pino, F. J. Garcia-Vidal, J. Feist, Exploiting vibrational strong coupling to make an optical parametric oscillator out of a Raman laser. Phys. Rev. Lett. 2016, 117, 277401.

23. J. Flick, M. Ruggenthaler, H. Appel, A. Rubio, Atoms and molecules in cavities, from weak to strong coupling in quantum-electrodynamics (QED) chemistry. Proc. Natl. Acad. Sci. USA 2017, 114, 3026-3034.

24. J. Galego, F. J. Garcia-Vidal, J. Feist, Many-molecule reaction triggered by a single photon in polaritonic chemistry. Phys. Rev. Lett. 2017, 119, 136001.

25. L. A. Martínez-Martínez, R. F. Ribeiro, J. CamposGonzález-Angulo, J. Yuen-Zhou, Can ultra strong coupling change ground-state chemical reactions? ACS Photonics 2018, 5, 167-176.

26. R. F. Ribeiro, L. A. Martínez-Martínez, M. Du, J. CamposGonzalez-Angulo, J. Yuen-Zhou, Polariton chemistry: controlling molecular dynamics with optical cavities. Chem. Sci. 2018, 9, 6325-6339. 
27. J. Galego, C. Climent, F. J. Garcia-Vidal, J. Feist, Cavity Casimir-Polder forces and their effects in ground-state chemical reactivity. Phys. Rev. X 2019, 9, 021057.

28. S. Kéna-Cohen, J. Yuen-Zhou, Polariton chemistry: Action in the dark. ACS Cent. Sci. 2019, 5, 386-388.

29. J. Yuen-Zhoua, V. M. Menonb, Polariton chemistry: Thinking inside the (photon) box. Proc. Natl. Acad. Sci. USA 2019, 116, 5214-5216.

30. J. Campos-Gonzalez-Angulo, R. F. Ribeiro, J. Yuen-Zhou, Resonant enhancement of thermally-activated chemical reactions via vibrational polaritons. 2019, arXiv preprint arXiv:1902.10264 [physics.chem-ph].

31. A. Mandal, P. Huo, Investigate new reactivities enabled by polariton photochemistry. 2019, ChemRxiv preprint. https://doi.org/10.26434/chemrxiv.8220875.v1.

32. F. Hernández, F. Herrera, Multi-level quantum Rabi model for anharmonic vibrational polaritons. 2019, arXiv preprint arXiv:1906.04374v1 [physics.chem-ph].

33. H. Hiura, A. Shalabney, J. George, Vibrational ultra strong coupling of water and ice, submitted to Angew. Chem. Int. $E d$.

34. Strictly speaking, "polariton chemistry" and "polaritonic chemistry" are distinguished as follows: the former is referred as to chemistry when the system is excited with real light and a polariton is formed, whereas the latter is chemistry in the ground state under vibrational light-matter coupling.

35. C. Ciuti, G. Bastard, Quantum vacuum properties of the intersubband cavity polariton field. Phys. Rev. B 2005, 72, 115303.

36. S. J. Bosman, M. F. Gely, V. Singh, A. Bruno, D. Bothner, G. A. Steele, Multi-mode ultra-strong coupling in circuit quantum electrodynamics. npj Quantum Information 2017, 3,46 .

37. J. Casanova, G. Romero, I. Lizuain, J. J. García-Ripoll, E. Solano, Deep Strong coupling regime of the JaynesCummings model. Phys. Rev. Lett. 2010, 105, 263603.

38. X. Cao, J. Q. You, H. Zheng, F. Nori, A qubit strongly coupled to a resonant cavity: asymmetry of the spontaneous emission spectrum beyond the rotating wave approximation. New J. Phys. 2011, 13, 073002.

39. P. Forn-Díaz, J. J. García-Ripoll, B. Peropadre, J.-L. Orgiazzi, M. A. Yurtalan, R. Belyansky, C. M.Wilson, A. Lupascu, Ultrastrong coupling of a single artificial atom to an electromagnetic continuum in the nonperturbative regime. Nat. Phys. 2017, 13, 39-43.

40. F. Yoshihara, T. Fuse, S. Ashhab, K. Kakuyanagi, S. Saito, K. Semba, Superconducting qubit-oscillator circuit beyond the ultrastrong-coupling regime. Nat. Phys. 2017, $13,44-47$.

41. S. Gambino, M. Mazzeo, A. Genco, O. Di Stefano, S. Savasta, S. Patanè, D. Ballarini, F. Mangione, G. Lerario, D. Sanvitto, and G. Gigli, Exploring light-matter interaction phenomena under ultrastrong coupling regime. ACS Photonics 2014, 1, 1042-1048.
42. L. C. Andreani, Strong Light-Matter Coupling: From Atoms to Solid-State Systems. A. Auffèves, D. Gerace, M. Richard, S. Portolan, M. França Santos, L.-C. Kwek, C. Miniatura Eds. (World Scientific Publishing Co. Pte. Ltd., Singapore, 2014) Chapter 2, pp.37-82.

43. N. E. Mavromatos, D.V. Nanopoulos, Quantum mechanics in cell microtubules: Wild imagination or realistic possibility. Adv. Struct. Biol. 1998, 5, 283-318.

44. S. G. Boxer, Stark realities. J. Phys. Chem. B 2009, 113, 2972-2983.

45. S. D. Fried, S. Bagchi, S. G. Boxer, Extreme electric fields power catalysis in the active site of ketosteroid isomerase. Science 2014, 346, 1510-1514.

46. S. D. Fried, S. G. Boxer, Measuring electric fields and noncovalent interactions using the vibrational Stark effect. Acc. Chem. Res. 2015, 48, 998-1006.

47. J. Yuen-Zhou, S. K. Saikin, T. Zhu, M. C. Onbasli, C. A. Ross, V. Bulovic, M. A. Baldo, Plexciton Dirac points and topological modes. Nat. Comm. 2016, 7, 11783.

48. D. S. Dovzhenko, S. V. Ryabchuk, Yu. P. Rakovich, I. R. Nabiev, Light-matter interaction in the strong coupling regime: configurations, conditions, and applications. Nanoscale 2018, 10, 3589-3605.

49. A. C. Aragonès, N. L. Haworth, N. Darwish, S. Ciampi, N. J. Bloomfield, G. G. Wallace, I. Diez-Perez, M. L. Coote, Electrostatic catalysis of a Diels-Alder reaction. Nature, 2016, 531, 88-91.

50. M. Aspelmeyer, T. J. Kippenberg, F. Marquardt, Cavity optomechanics. Rev. Mod. Phys. 2014, 86, 1391.

51. J. M. Pitarke, V. M. Silkin, E. V. Chulkov, P. M. Echenique, Theory of surface plasmons and surface-plasmon polaritons. Rep. Prog. Phys. 2007, 70, 1-87.

52. G. B. Arfken, H. J. Weber, Mathematical Methods for Physicists. 6th ed. (Elsevier Academic Press, London, 2005) Chapter 5.

53. M. Cockett, G. Doggett, Maths for Chemist Volume 2, Power Series, Complex Numbers and Linear Algebra (The Royal Society of Chemistry, Cambridge, 2003) Chapter 1 and 3 .

54. If using Eq. 2, the relative activation energy $E_{ \pm} / E_{0}$ may be expressed as follows:

$$
\frac{E_{ \pm}}{E_{0}} \approx\left(1 \pm \frac{1}{2} \frac{\Omega_{\mathrm{R}}}{\omega_{0}}\right)^{2}
$$

55. P. Hänggi, P. Talkner, M. Borkovec, Reaction-rate theory: fifty years after Kramers, Rev. Mod. Phys. 1990, 62, 251.

56. H. Eyring, The activated complex in chemical reactions. $J$. Chem. Phys. 1935, 3, 107.

57. If using Eq. 2, the Arrhenius type $\kappa_{ \pm} / \kappa_{0}$ may be expressed as follows:

$$
\frac{\kappa_{ \pm}}{\kappa_{0}} \approx \exp \left[\left(-\frac{E_{0}}{k_{\mathrm{B}} T}\right)\left\{\left(1 \pm \frac{1}{2} \frac{\Omega_{\mathrm{R}}}{\omega_{0}}\right)^{2}-1\right\}\right]
$$

58. If using Eq. 2, the Eyring-Polanyi type $\kappa_{ \pm} / \kappa_{0}$ may be expressed as follows:

$\frac{\kappa_{ \pm}}{\kappa_{0}} \approx\left(1 \pm \frac{1}{2} \frac{\Omega_{\mathrm{R}}}{\omega_{0}}\right) \exp \left[\left(-\frac{E_{0}}{k_{\mathrm{B}} T}\right)\left\{\left(1 \pm \frac{1}{2} \frac{\Omega_{\mathrm{R}}}{\omega_{0}}\right)^{2}-1\right\}\right]$ 